\title{
Editorial
}

\section{Always Caring, Especially When Cure is not Possible - An Essential Tenet of Neonatal Care}

\section{John V. Hartline, MD}

Journal of Perinatology (2002) 22, 183 DOI: 10.1038/sj/jp/7210693

Neonatology, like invasive cardiology, organ transplantation and other aggressive, technology-dependent areas of heath care arose in an era when the focus of all heath care was on cure. Data on effectiveness stressed survival (or its converse, mortality) with little regard for morbidity. The roles of medicine and its related professions were to seek out illnesses and smite them with the swords of technology. The vernacular of medicine was that of war - cure was winning; death was the ultimate loss. Somehow, the realities that we all must die and that there are situations when the weapons of the war on disease yield burdens of suffering or consequences of interventions exceeding the intended benefits were left out of discussions, as if they were undiscovered. Yet, at the bedside, patients, families, and many caregivers endured the long-suffering associated with half-way technologies and many patients and families carried out the end of life rituals after being abandoned by a cure-focused health care system.

To fill the void, hospice programs arose to meet the needs of dying patients and their families. Initially, eligibility for hospice required medical certification that the illness was terminal and death was imminent. Participation required abandonment of any hope for survival or cure and acceptance only of measures directed toward comfort. The results: too little and most often too late. Over time and with the guidance of many health professionals who studied severe illness at the end of life, caring elements of the hospice movement have modified all health care - resulting in improvements in the inpatient environment (single-patient rooms, for example), incorporation of family, recognition of the essential roles for social workers and clergy, coordination of hospital-based and communitybased care, and transition of many patients' care to the home setting.

Some neonatologists came early to the recognition of unduly burdensome or futile care and to the reality that some infants dying in newborn units were allowed to do so. ${ }^{1,2}$ Unfortunately, this early recognition led to reaction rather than acceptance. For many years

Michigan State University/Kalamazoo Center for Medical Studies, Bronson Methodist Hospital, 252 E. Lovell, \#2230, Kalamazoo, MI, USA.

Address correspondence and reprint requests to John V. Hartline, MD, Michigan State University/Kalamazoo Center for Medical Studies, Bronson Methodist Hospital, 601 John St., Box 41, Kalamazoo, MI 49007, USA thereafter, discussions stressed the need to prevent inappropriate forgoing of possibly useful interventions. But, suffering infants with clearly terminal conditions did not disappear and many had lifeprolonging interventions imposed on them with little or no potential for improvement and without informed consent from the parents. More discussions arose on subjects such as "limits of viability," "criteria for resuscitation in the delivery room," and "withholding or withdrawing life prolonging interventions." The needs of education and resources to deal with appropriateness of care, withholding or withdrawing life-extending measures, and support of the dying infant and his/her family have surfaced in every neonatal intensive care unit. The Neonatal Resuscitation Program has incorporated criteria for not applying neonatal resuscitation in the delivery room. These criteria simultaneously require the initiation of palliative care for the infant, the family, and the professional staff. As ventilator management or parenteral nutrition, palliative care is a necessary competence for neonatologists and the professionals with whom they work.

In this issue, Anita Catlin and Brian Carter ${ }^{3}$ have outlined the essentials of a palliative care program. Their approach emphasizes the importance of understanding the needs of the dying or suffering individual patient, of discerning and responding to the culturally affected needs of families, and of supporting the health professional staff. Their comprehensive discussion evolved from interviews with numerous and varied professionals, from reviews of existing protocols and procedures, and from application of the Delphi Methodology to meld differing views into a consensus statement. Application of their recommendations should be helpful to all who confront the challenges of newborn intensive care. Whether used as a guide for formally establishing a hospice unit or consultation service, or as guidance when confronted with the individual in need of palliative care, this comprehensive discussion should lead to competence in an often needed and most difficult area within neonatology.

\section{References}

1. Duff RS, Campbell AG. Moral and ethical dilemmas in the special care nursery. N Engl J Med 1973;289(17):890-4

2. Duff RS, Campbell AG. On deciding the care of severely handicapped or dying persons with particular reference to infants. Pediatrics 1976;57(4):487-93.

3. Catlin A, Carter B. Creation of a neonatal end-of-life palliative care protocol. J Perinatology 2002;22(3):184-95. 